\title{
Reconstrucción mamaria en una paciente con quemadura por abrasión
}

Dr. Miriam Aicardi

Cirujana Plástica, Estética y Reparadora

Miembro Titular de la Sociedad Boliviana de Cirugía Plástica,

FILACP, IPRAS,

Dra. Gabriela Antelo Pomacusi

Cirujana General

Miembro Titular de la Sociedad Boliviana de Cirugía General, Hospital Universitario Japonés
Dr. Marco Antonio Vaca Díez

Anestesiólogo

Miembro Titular de la Sociedad Boliviana de Anestesiología, Hospital Villa $1^{\circ} \stackrel{\text { de Mayo }}{ }$

Ana María Párraga

Enfermera - Instrumentadora

Hospital de Niños

Miriam Aicardi

Calle Charcas № 862 (entre 1er. y 2do. Anillo)

E-mail: maicardi88@icloud.com

Santa Cruz de la Sierra-Bolivia

\section{RESUMEN}

\section{Palabras claves:}

Quemadura por abrasión, reconstrucción mamaria, colgajo, pedículo superior.

Se recibió una paciente (sexo femenino, 18 años) con quemaduras por abrasión. Comprometió tórax, abdomen, codos, miembros inferiores y mejilla izquierda. Evoluciona con adherencia completa de mama izquierda a abdomen. Distancia areola-pezón a ombligo: 4cm.

Se planifica la reconstrucción mamaria y toraco-abdominal.

Se reconstruye la mama con un colgajo dermo-graso a Pedículo superior.

Se reconstruye la pared del abdomen mediante autoinjertos de piel.

\section{ABSTRAC}

\section{Key words:}

Burn by abrasion, breast reconstruction, flap, high pedicle.

We received a patient (female, 18 years old) with burn by abrasion.

It took thorax, abdomen, elbows, legs and the left cheek.
The burn develop a big adhesion between the left breast and the abdomen.

The distance between the areola-nipple - umbilicus were $4 \mathrm{~cm}$.

The breast reconstruction was prepared by a flap with a high pedicle.

The breast flap was done and the abdomen was fixed by skin graft.

\section{INTRODUCCIÓN}

Se trata de un paciente de sexo femenino, de 18 años de edad que concurre por primera vez a la consulta por presentar graves secuelas de quemadura por abrasión, de dos años de evolución, que no fueron tratadas.

Hace dos años la paciente sufre un accidente de tránsito, donde fue arrastrada sobre el asfalto por un vehículo y luego arrojada en un terreno con lodo.

Luego de unas horas fue encontrada por los vecinos del lugary asistida en un Centro privado de salud.

Se le diagnostica:

TEC y fractura de miembro inferior izquierdo 
Quemaduras por abrasión (18\% SCQ, localización: tórax, abdomen, muslo y rodilla derechos, ambos codos y mejilla derecha)

Permaneció en UTI un mes, fue sometida a osteosíntesis de fémur izquierdo y luego de ser dada de alta por el TEC, se le efectuaron algunas limpiezas quirúrgicas en el área de sus quemaduras por abrasión.

Fue dada de alta y concurría a una enfermería próxima a su casa para curaciones de las áreas quemadas, sin anestesia ni sedación.

Luego de 3 meses, el área cruenta de la mama izquierda se había adherido completamente al abdomen, estando el complejo areola-pezón a $4 \mathrm{~cm}$ de distancia del ombligo, lo cual impedía que la paciente camine erguida.

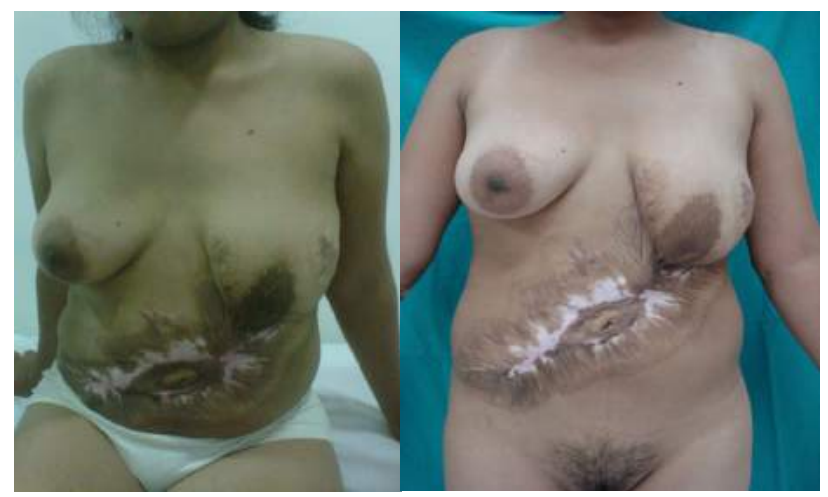

Después de tres meses, la paciente presentaba este cuadro

\section{MATERIAL Y MÉTODO}

Analítico, Longitudinal, Prospectivo, Experimental.

Planteamiento quirúrgico:

Plan A: Despegamiento mamario más colgajo.

Plan B: Despegamiento mamario más colgajo e implante mamario, si fuese necesario dar más volumen a esta mama.

Plan C: Despegamiento mamario más colgajo de dorsal ancho, si la mama una vez liberada del abdomen presente un volumen demasiado pequeño.

Se aplicó el plan A.

Procedimiento Quirúrgico:

Paciente bajo anestesia general.

Se marca previamente y se toma la distancia entre la línea medio clavicular - pezón (lado derecho).

Se marca surco submamario.
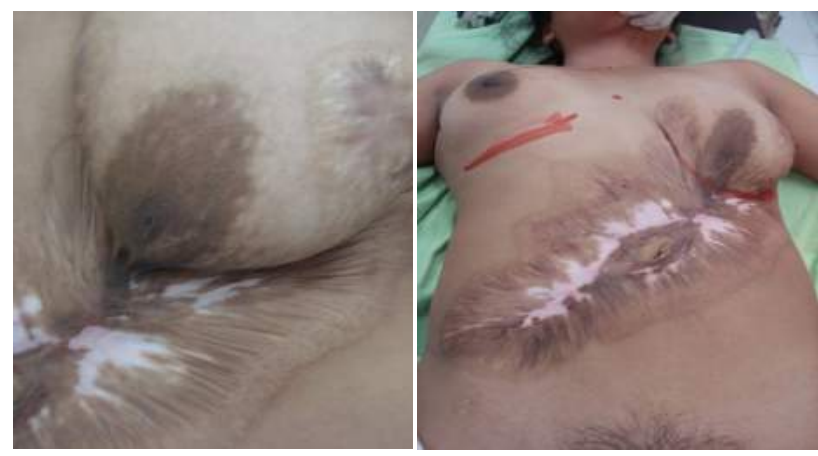

Marcación de surco submamario

Se realiza incisión en la unión de la mama con el abdomen y se decola.

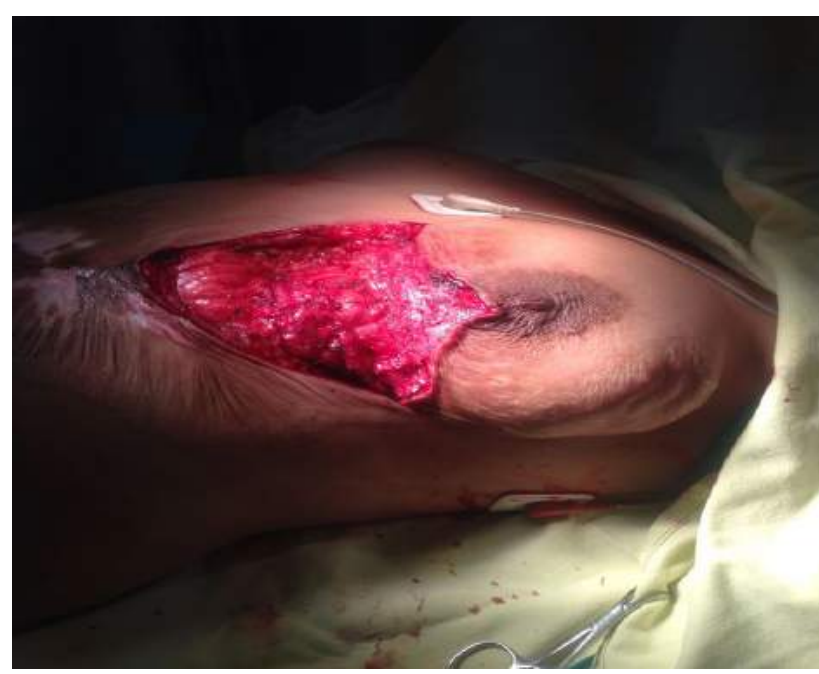

Decolamiento de la mama

Se confecciona un colgajo dermoglandular a pedículo superior.

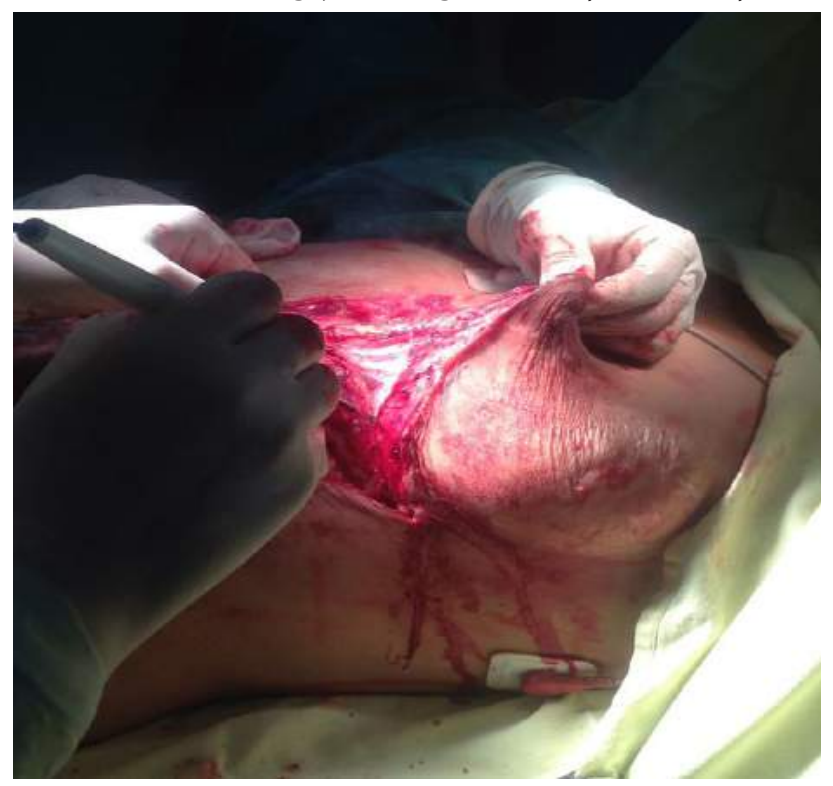

Colgajo dermoglandular 
El colgajo es totalmente liberado.

Se fija el colgajo en su porción más alta en el polo superior.

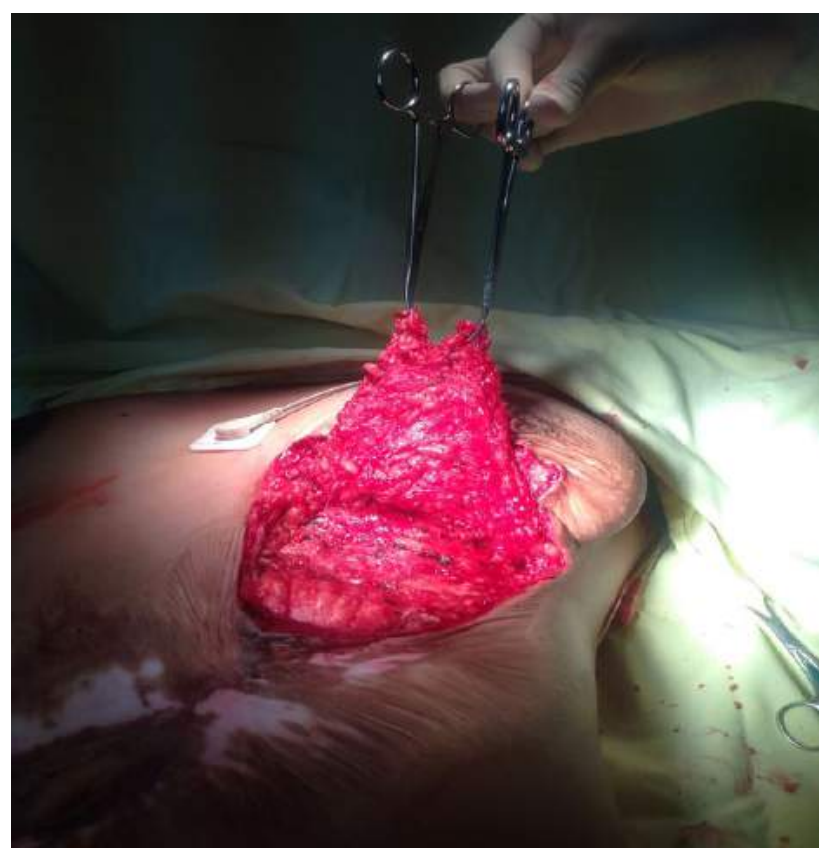

Colgajo liberado

Posteriormente se procede al cierre de la mama, uniendo los puntos B y C en la porción media del surco submamario.

La nueva posición de la areola se determina teniendo en cuenta la mama contralateral.

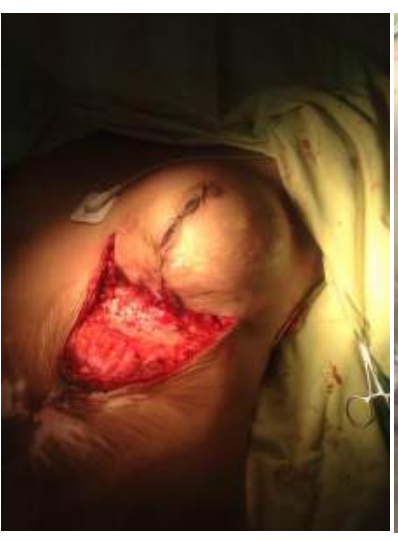

Cierre de la mama

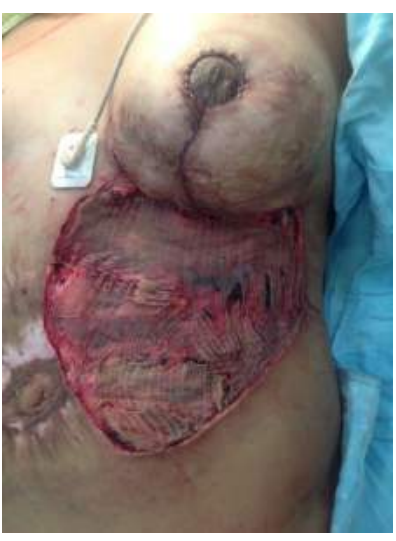

Cobertura con autoinjerto de piel
Se cubre el área cruenta del abdomen con autoinjerto de piel.

\section{RESULTADOS}

Satisfactorios desde el punto de vista estético y funcional para la paciente.
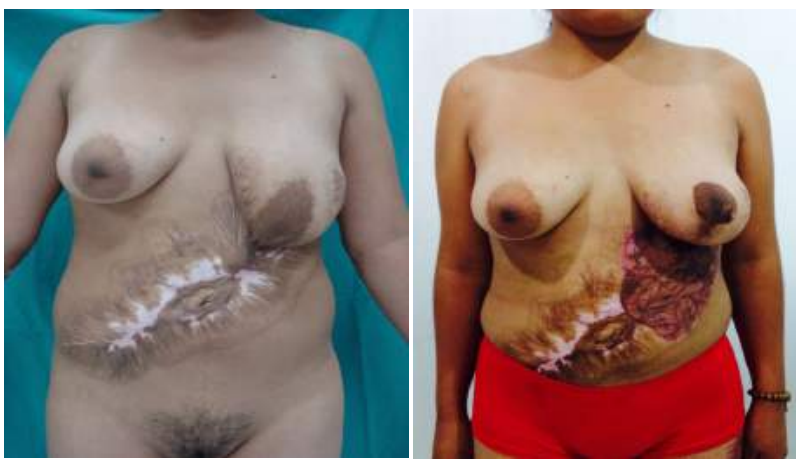

Pre operatorio

Postoperatorio (luego de 3 meses)

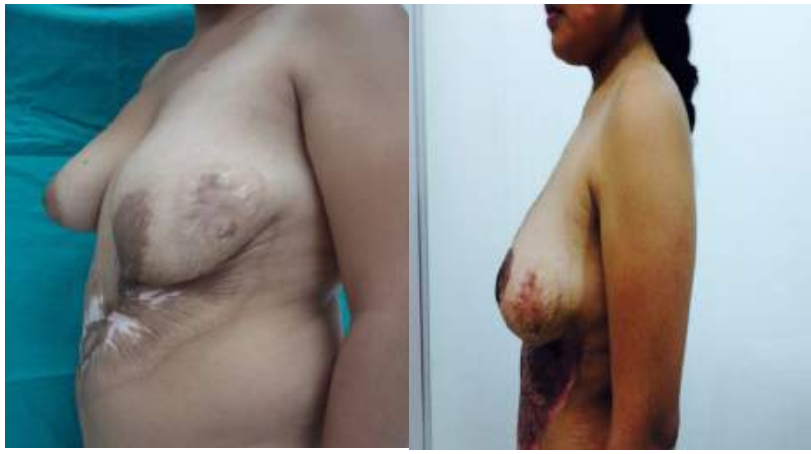

Vista lateral preoperatorio Vista lateral postoperatorio

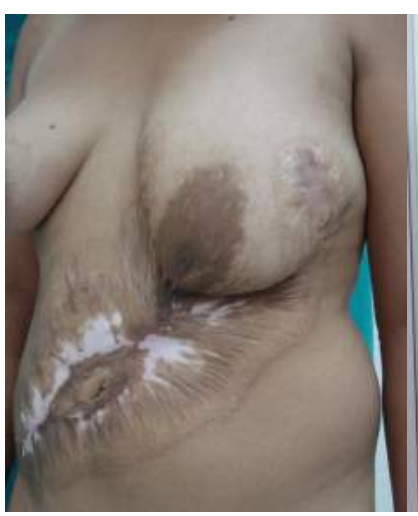

Perfil 3/4 preoperatorio

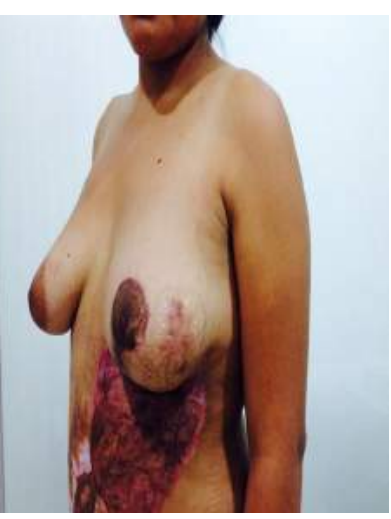

Perfil 3/4 postoperatorio

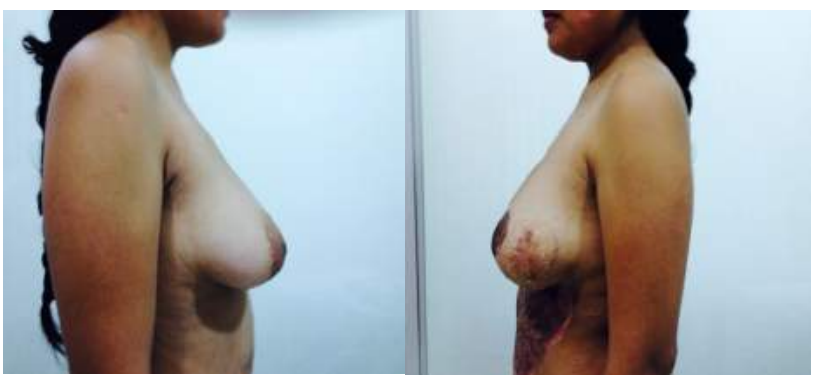

Mama derecha sana

Mama izquierda reconstruida 


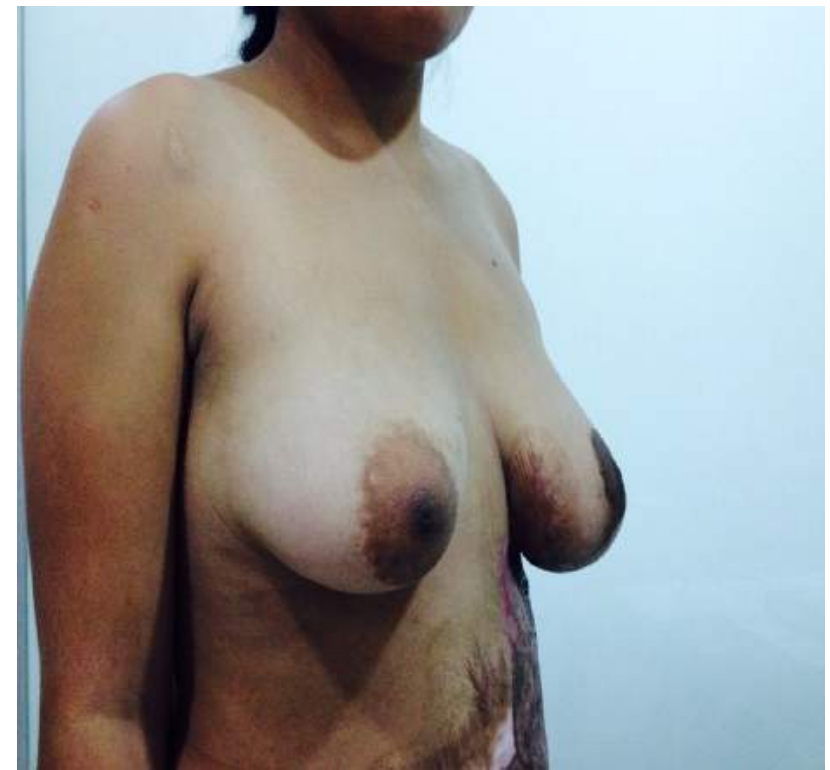

Perfil mama derecha sana

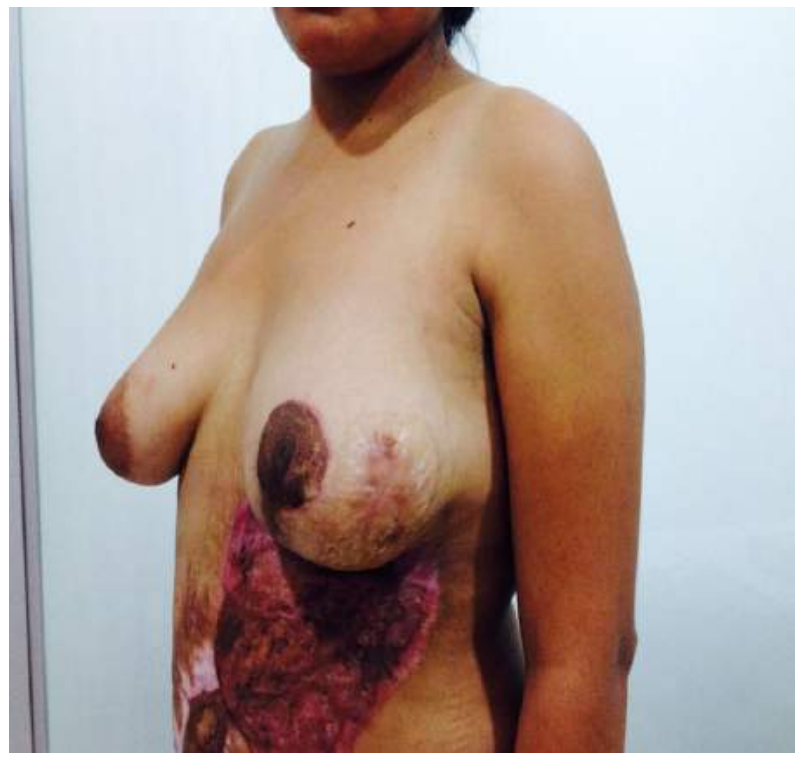

Perfil mama izquierda reconstruida

Ambas tienen similar proyección y forma

\section{DISCUSIÓN Y CONCLUSIÓN}

Consideramos que la planificación quirúrgica fue la más adecuada de las tres opciones presentes: reconstrucción mamaria a pedículo inferior.

\section{AGRADECIMIENTO}

A la Sra. Nina Abendroth Ibbeken por su ayuda solidaria, sin la cual no hubiera sido posible esta cirugía.

\section{BIBLIOGRAFÍA}

1. Berrocal Revueltas, M. (2014). Las Cuatro Estaciones de las Mamas. Evaluación, diseño y aplicación de procedimientos quirúrgicos. Bogotá, Colombia: Impresión Médica.

2. Coiffman,F. (2008). Cirugía Plástica, Reconstructiva y Estética. Senos-Tronco- Miembros Inferiores. (3* edic). Tomo IV. Primera parte. Sección II: Mamas (p.2813). Caracas, Venezuela: Amolca.

3. Herndon, D. N. (2009). Tratamiento Integral de las Quemaduras. ( $3^{\star}$ edic). Barcelona, Espana: Elsevier Masson.

4. Ribeiro, L. (2009). Pedículos en Mamoplastia. Atlas y Texto. Caracas, Venezuela: Amolca.

5. Spear, S.L. (2008). Cirugía de la Mama. Principios y Arte. ( $2^{\star}$ edic.). Tomo 2. Caracas, Venezuela: Amolca.

6. Testut, L. y Jacob, O. (1985). Anatomía Topográfica. Tomo. Libro Cuarto Tórax (p. 753). Barcelona, España: Salvat Editores. 